\title{
Marching Bear: An Interface System Encouraging User's Emotional Attachment and Providing an Immersive Experience
}

\author{
Nagisa Munekata, Takanori Komatsu, and Hitoshi Matsubara \\ Future University-Hakodate \\ 116-2 Kamedanakano, Hakodate 041-8655, Japan \\ $\{$ g3106012, komatsu, matsubar\} @fun.ac.jp \\ http://www. fun.ac.jp
}

\begin{abstract}
We developed an interface system called "Marching Bear," where the robot controller's motions were reflected in the motions of certain characters appearing on a computer display. We then conducted a simple experiment to investigate the effectiveness of this interface system to see whether users felt that this interface system was enjoyable. We used a questionnaire and conducted an observation to determine whether users could direct the robot's arms in such a way as to make the character walk. We also investigated the effectiveness of our interface system with two displays, one large, the other small. The results were that most participants felt this system was enjoyable and that they could direct the robot's arms in the way needed to make the character walk. The larger display had a significantly stronger effect on the participants' emotional aspects; they felt that the character had some emotions and that they could communicate with the character well. Therefore, the interface system, Marching Bear, was not only enjoyable for users because of the robot controls, but also this type of interface system may become a users' companion or be perceived as an independent character.
\end{abstract}

Keywords: interface system, emotional aspect, independent character, companion.

\section{Introduction}

Many researchers have developed various video game controllers based on the diversification of video game content and the interest in creating more immersive gameplay. The "Wii Remote" developed by Nintendo [1], "Tatacon" developed by NAMCO [2], and the "EyeToy USB Camera" developed by Sony Computer Entertainment of Europe [3] are three examples of such controllers. The Wii Remote is a remote game controller based on motion sensing technology, and it is specifically designed for many different kinds of video games, including sports, fighting, racing, and shooting games. Tatacon is a drumstick-shaped video game controller where users hold the sticks to beat a drum in time with the music. The EyeToy USB Camera is normally placed on top of a display, and this can detect the bodily movements of 
users who play a video game in front of it. These intuitive controllers were developed with the goal of enabling everyone to enjoy playing video games, regardless of their age, gender, or experience at video games. However, at first, it takes some time for users to get accustomed to operating these controllers intuitively so that they pay attention to the controller itself and not the game. The users do not really feel a sense of immersion with the game as long as they are struggling with operating the controller (Figure 1-A).

To solve this problem, some studies have developed a new type of controller using a robot, a so-called robotic user interface (RUI) [4] [5] [6] (Figure 1-B). The RUI is designed to make users feel as if there are no borders between a robot in the real world and a character in the virtual world. A character in the virtual world can be controlled by the motions of a robot used as a controller in the real world. For example, Koizumi et al. developed a hand puppet RUI; its robot controller has a potentiometer and a triaxial acceleration sensor in the robot, which is operated by a user, to detect its motions [7]. This RUI makes the robot in the real world start to vibrate to inform the user that a character has hit one or more obstacles in the virtual world. However, this system was basically designed to satisfy the users' operationalities and functionalities. Therefore, immersive experiences of users are up to the quality of the game content.

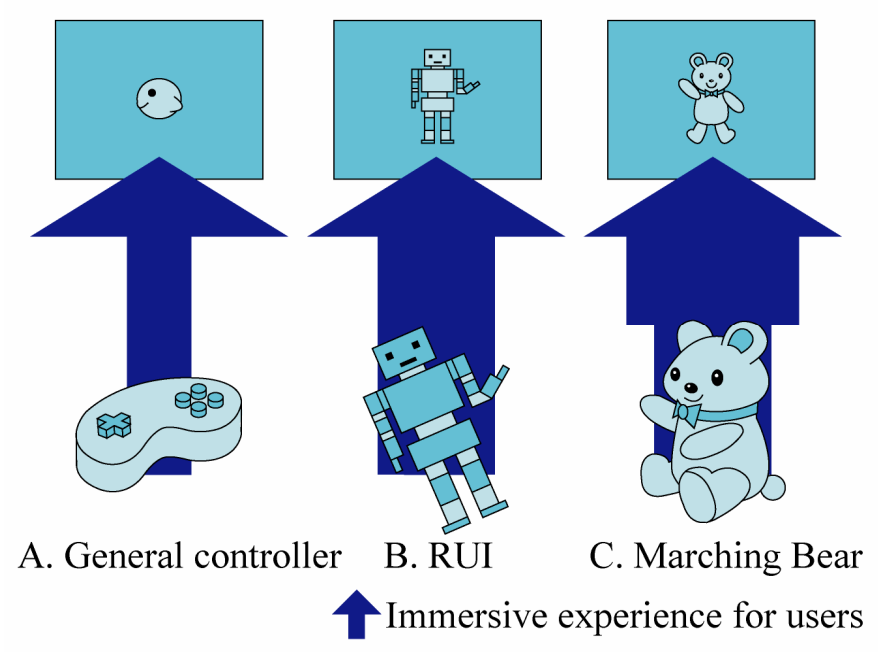

Fig. 1. Immersive experience for users

We wanted to develop a system with not only operationality and functionality but also one where users develop a sense of immersion regardless of the game content. We created an interface system using a robot called "Marching Bear." This interface was principally designed to make the user interested in the controlling operations. We hypothesized that the users' interest in the operations would lead them to feel somewhat of an emotional attachment to the interface system itself, and that these feelings would eventually create a strong immersive experience for not only the interface system but also the game content. 


\section{System Overview of Marching Bear}

\subsection{Robot Controller}

In our Marching Bear, a stuffed animal like robot is utilized as a controller for users (Figure 2). This robot is a consumer product created by the Iwaya Corporation [7], and it was originally developed to be a communication terminal that connects with IP telephones [8]. The joint configuration of this robot is designed to be similar to a person's upper body: each arm has 2 degrees-of-freedom (DOFs) in the shoulder joint, and the neck has 2 DOFs in a pan-tilt joint. Each joint consists of a pair of servomotors and a joint angle sensor so that the users can directly move these joints using their own hands, and the joint angle values can be measured when they move. This robot controller was designed like a teddy bear so that users would have a positive impression based on the cute appearance and that this would motivate users to interact with the controller.

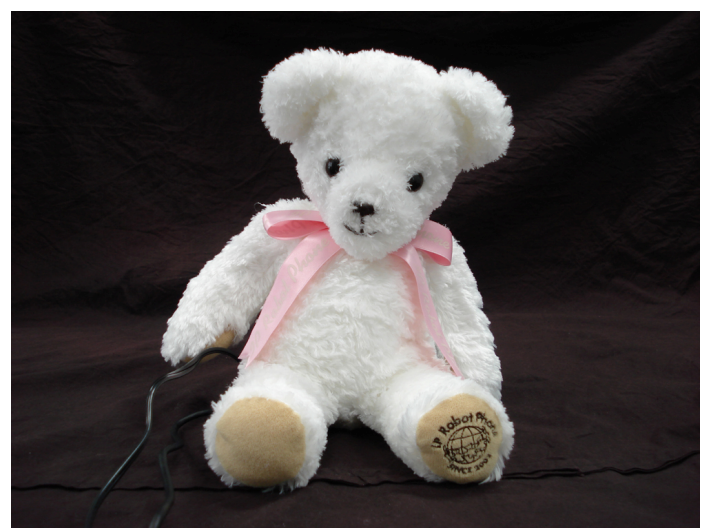

Fig. 2. IP Robot Phone (by the Iwaya Corporation)

\subsection{System Configuration}

The left figure in Figure 3 shows the overview of the interface system, Marching Bear. This system consists of the robot controller and a virtual bear-like character that appears on a computer display.

Users hold the stuffed animal robot as a controller, and the character on the computer display reflects the users' movements of the robot controller. For example, if the user raises the controller's right-arm, the character's right arm moves. If the user makes the controller's head nod, the character also simultaneously nods its head.

In this system, the virtual character can walk in the virtual world on the computer display only when a user alternates moving the robot controller's arms - as if he or she were leading a toddler by the hands as part of teaching them how to walk. Now think about how we teach toddlers to walk; we do not grab their legs directly; instead, 

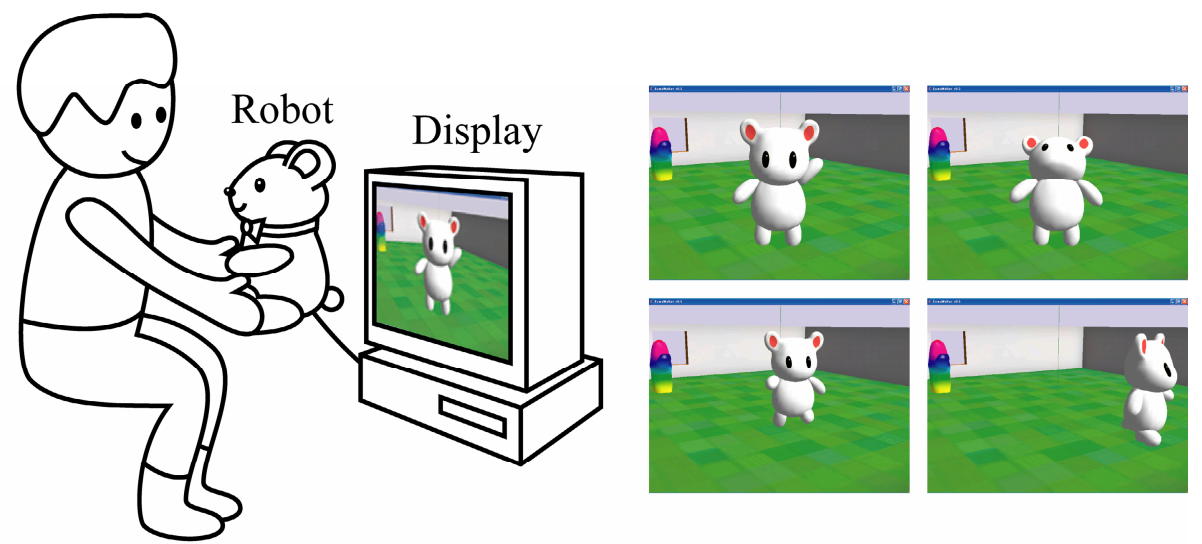

Fig. 3. Interface system, Marching Bear (left), and virtual character on the computer display (right)

we get them to walk by leading them by the hands. Thus, we believe that the users' behavior of moving the robots' arms to make it walk indicate that the users regard this robot as an independent character. In this case, these users would have some emotional attachment and feel an immersive experience. However, if users regard this as just a tool for controlling the character, they would grab the robot's legs instead. We believe that grabbing the robot's arms to make it walk, which is an action similar to teaching toddlers to walk, is important for getting users to enjoy controlling the robot.

\section{Experiment}

\subsection{Overview}

We conducted a simple psychological experiment to investigate the effectiveness of this interface system and whether or not users felt that this interface system was enjoyable. We used a questionnaire and conducted an observation to see whether users could move the robot's arms to make the character walk. We also wanted to investigate the effectiveness of different sizes of the virtual character by using a large display and a small one.

\subsection{Participants}

The participants were 20 Japanese university students and university staff members (12 men and 8 women, $22-45$ years old). All participants had never seen this interface system and an IP Robot Phone. The participants were randomly assigned to the following two groups, i.e., a between-subjects design was used (Figure 4). 

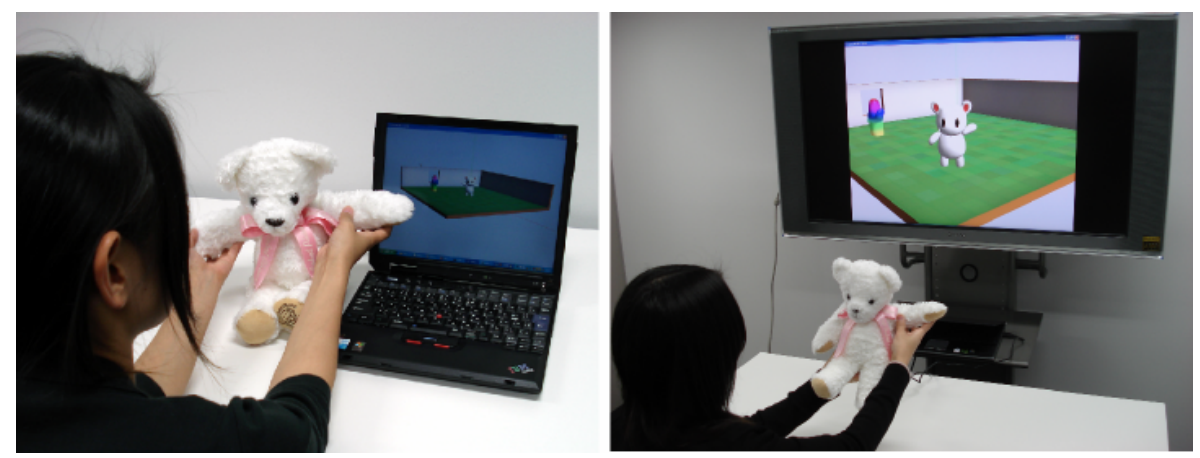

Fig. 4. Experimental Scene (Left: Small character group, right: Large character group)

- Small character group (10 participants): These participants experienced an interface system with a virtual character in a display that was smaller than the actual robot controller. In this experiment, the virtual character was displayed on a laptop PC's screen, which was 13.3 inches (WXGA, 1280 x 800 pixels), so the actual height of the virtual character was from about $1.5 \mathrm{~cm}$ (at the back of the virtual world) to $4.5 \mathrm{~cm}$ (at the front of the virtual world).

- Large character group (10 participants): These participants experienced a system with a virtual character in a display that was much more similar in size to the actual robot controller. In this experiment, the virtual character was displayed on an LCD monitor's screen, which was 46 inches (WXGA, 1920 x 1080 pixels), so the actual height of the virtual character was from about $5 \mathrm{~cm}$ (at the back of the virtual world) to $15.0 \mathrm{~cm}$ (at the front of the virtual world).

1 You could understand immediately how to make the bear walk.

2 You felt sorry for the bear when it hit the wall.

3 You could move the bear walk around you wanted it to.

4 You could control the bear's movements completely and intuitively.

5 The bear has some kinds of emotions.

6 You felt that you could communicate with the bear well

7 The movements of the bear were cute.

8 You enjoyed making the bear move.

9 You wanted to play with the bear more.

10 You felt that the bear hated to walk out of this room.

11 You wanted to take the bear home.

12 You felt uncomfortable with this stuffed animal robot.

Fig. 5. Questionnaire Used in the Experiment 


\subsection{Procedure}

First, an experimenter gave the instruction, "Please feel free to touch and move this stuffed animal robot for ten minutes. If you have any questions about this system, I will answer them after the first three minutes." The experimenter did not mention that moving the controller's arms back and forth makes the character walk. In addition, the experimenter did not inform the participants that the name of this interface system was the "Marching Bear." After a ten-minute trial, all participants were asked to fill in the questionnaire shown in Figure 5. This questionnaire was designed with a fivepoint likert scale (one point was the worst assessment, and five points was the best). Finally, all participants were interviewed by the experimenter about their impression of playing with the Marching Bear.

\section{Results}

\subsection{Comparison of the Participants Behaviors}

The results of the observation of the users' behaviors indicated that all participants succeeded in making the Marching Bear walk within ten minutes. Five of the participants (small character group: two, large character group: three) succeeded it within three minutes without any help from the experimenter. At first, these five participants focused on moving the robot's legs to make the virtual character walk, and they noticed that the character did not react. Then, they shifted their attention from the legs to the arms, and they eventually noticed how to make this character walk. They reported in their interviews after the experiment that they thought that the character might be able to walk when they saw it at the beginning of the experiment, so they tried to make it walk.

The behaviors of the remaining 15 participants were nearly the same as the first five. At first, they also thought that the character might be able to walk, but they did not show the specific behaviors needed to make the character walk, e.g., they just hugged it or shook its hands. After the first three minutes, they asked the experimenter whether this character could walk or not. The experimenter answered, "It can walk." After this was stated, the participants immediately focused on moving the robot's legs, and eventually, they too noticed how to make the character walk.

\subsection{Comparison of the Participants' Questionnaires}

Figure 6 shows the results of the questionnaire of the two groups. The scores on Q7, "The movements of the bear were cute", and 08 , "You enjoyed making the bear move”, were over four points for both groups (the maximum was five). Thus, most participants of both groups enjoyed playing with the Marching Bear.

The results of an ANOVA revealed significant differences in the answers of four out of the 12 questions between the small character group and the large character group: Q3, "You could make the bear walk around as you wanted it to," Q5, "The 


\begin{tabular}{|c|l|c|c|c|}
\hline No & \multicolumn{1}{|c|}{ Question } & $\begin{array}{c}\text { Small } \\
\text { character }\end{array}$ & $\begin{array}{c}\text { Large } \\
\text { character }\end{array}$ & $\begin{array}{c}\text { Significant } \\
\text { differences }\end{array}$ \\
\hline 1 & Understand immediately & 2.0 & 1.9 & - \\
\hline 2 & Sorry when it hit the wall & 2.8 & 3.3 & - \\
\hline 3 & Make the bear walk as you wanted it to & 3.3 & 4.1 & $\mathrm{p}<.05$ \\
\hline 4 & Control the bear's movements intuitively & 2.8 & 3.1 & - \\
\hline 5 & The bear has emotions & 1.8 & 2.9 & $\mathrm{p}<.05$ \\
\hline 6 & Could communicate well & 2.1 & 2.8 & $\mathrm{p}<.10$ \\
\hline 7 & Movements were cute & 4.1 & 4.1 & - \\
\hline 8 & Enjoyed making the bear move & 4.2 & 4.4 & - \\
\hline 9 & Wanted to play more & 3.7 & 4.1 & - \\
\hline 10 & The bear hated to be out of this room & 2.7 & 3.0 & - \\
\hline 11 & Wanted to take the bear home & 3.4 & 3.7 & - \\
\hline 12 & Uncomfortable with this robot & 2.1 & 1.4 & $\mathrm{p}<.05$ \\
\hline
\end{tabular}

Fig. 6. Results of the questionnaires

bear has some kinds of emotions," Q6, "You felt that you could communicate withthe bear well," and Q12, "You felt uncomfortable with this stuffed animal robot." The results revealed that the participants in the large character group enjoyed their experience significantly more than the ones in the small character group. The comparisons of these four questions are described in the following sections.

\subsubsection{Q5 and Q6}

As already noted, the scores on Q5, “The bear has some kinds of emotions," for participants in the large character group were higher than the ones in the small character group. Specifically, the average score in the small character group was 1.8, while the one in the large character group was 2.9. The results of the ANOVA showed the differences between them were significant $\left(F(1,18)=7.51, \mathrm{p}<.05\left(^{*}\right)\right)$. In addition, for Q6, "You felt that you could communicate with the bear well," the average score in the small character group was 2.1, while the one in the large character group was 2.8 . The scores of Q6 were analyzed by a two-factor (character size: small or large) between subjects ANOVA. The results of the ANOVA showed significant tendencies between these scores $(F(1,18)=3.53, \mathrm{p}<.10(+))$. These results revealed that participants in the large character group regarded this robot and the virtual character as an independent character that had some emotions, more so than the ones in the small character group.

We thought that the reason for this low score in the small character group was caused by the fact that the size of the virtual character was too small compared to the robot controller. Actually, the height of the virtual character appearing on the computer display was from about 1.5 to $4.5 \mathrm{~cm}$, while the height of the robot controller was about $30 \mathrm{~cm}$. The participants could not understand why their movement of the robot was not clearly reflected in the character appearing on the display. 


\subsubsection{Q3 and Q4}

Q3, "You could make the bear walk around as you wanted it to," was a question about the operability of the Marching Bear, as was Q4, "You could control the bear's movements completely and intuitively.” Therefore, Q3 and Q4 were very similar. However, the results of the ANOVA revealed no significant difference in Q4 $(F(1,18)=0.77$, n.s. $)$, but a significant difference was found in Q3 $(F(1,18)=4.43$, $\mathrm{p}<.05(*))$ (Figure 7).
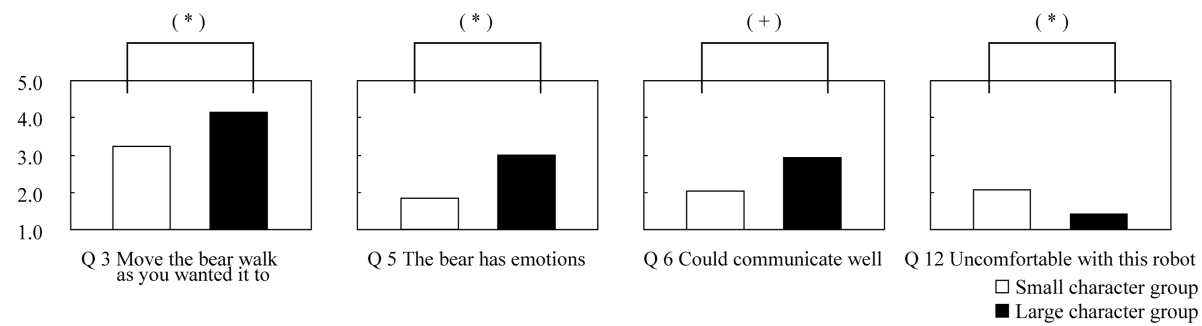

Fig. 7. Comparison of scores of questionnaires for small character group and large character group

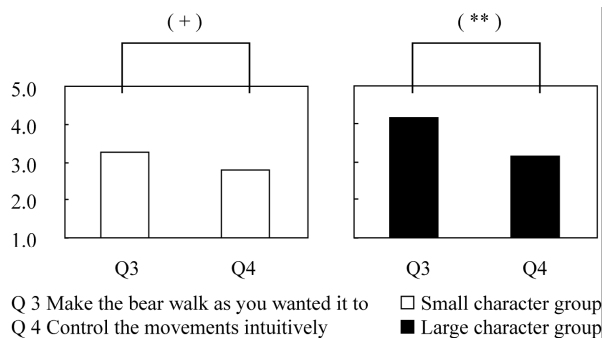

Fig. 8. Comparison of scores of questionnaires between Q3 and Q4 in each group

We expected some differences in understanding these two questions between the two groups. Thus, we conducted a statistical analysis to clarify these differences. The scores of Q3 and Q4 were analyzed by a two-factor (question: Q3 or Q4) within subjects ANOVA for each group. The results of the ANOVA showed a significant tendency $(F(1,9)=5.00, \mathrm{p}<.10(+))$ in the small character group and significant differences $(F(1,9)=11.25, \mathrm{p}<.01(* *))$ in the large character group (Figure 8). Thus, the participants in the large character group felt that they did not control the Marching Bear but made the Marching Bear walk just as they wanted it to.

We thought that this was because there were differences in utilized keywords between Q3 and Q4. In Q3, the major keywords were "make the bear walk" and "as you wanted it to", while in Q4, they were "control" and "intuitively." Therefore, the keywords in Q3 eventually evoked in the participants an interactive relationship between participants and the Marching Bear, and the keywords in Q4 evoked in them a unilateral relationship between the participants and the Marching Bear. 


\subsubsection{Q8 and Q12}

The scores of $\mathbf{0 8 , ~ " Y o u ~ e n j o y e d ~ m a k i n g ~ t h e ~ b e a r ~ m o v e , " ~ i n ~ b o t h ~ g r o u p s ~ w e r e ~ t h e ~}$ highest among all 12 questions. The average score in the small and large character groups was 4.2 and 4.4 point, respectively, with no significant difference between them $(F(1,18)=0.36$, n.s.). Both groups enjoyed playing with the Marching Bear. However, in Q12, "You felt uncomfortable with this stuffed animal robot," we found a significant difference between the scores in the small character group and the large character group: 2.1 and 1.4, respectively (Figure 7).

These results show that although the participants in the small character group enjoyed controlling the Marching Bear, they felt more uncomfortable with the stuffed animal robot than the participants in the large character group. This means that the participants in the former group seemed to enjoy controlling this robot as "just a controller." They perhaps felt some annoyance with utilizing a stuffed animal robot just to control a character on a display, and they would have preferred utilizing a more typical game controller.

\subsection{Summary}

We observed the following common phenomena in the small and large character groups based on the results of their questionnaires.

- The participants in both groups (small and large character) discovered how to make the character walk within ten minutes.

- The participants in both group first focused on moving the robot's legs to make the virtual character walk.

- The participants felt that the Marching Bear was cute, and they reported that they enjoyed making this character move around.

The results of the questionnaire from participants in both groups demonstrated the following.

- Small character group: The participants in this group saw the Marching Bear as part of a unilateral control relationship more so than the participants in the other group did.

- Large character group: The participants felt a stronger emotional attachment and a more immersive experience than the participants in the small character group. They felt that the character had some emotions and that they could communicate with the character well.

Thus, we found that the interface system Marching Bear succeeded in making users interested in the controlling operations. Displaying the virtual character on a larger screen, which could make the participants readily understand their spontaneous actions, would be effective for them to get interest in this system. We thought that these behaviors of the participants might lead to them sustaining their emotional attachment and sense of immersion with this interface system, as we had designed it to do. 


\section{Discussion}

\subsection{Effectiveness of Character's Size}

Most participants in the small character group answered that the Marching Bear did not have any emotions but that they enjoyed playing with it as a unilateral controller, much like a typical video game controller. This relationship between the general controller and video games was observed. In this case, when their operating virtual character was suddenly hit by some flying obstacles, most players would react to avoiding these obstacles in the real world. Here, they had some empathy for the virtual character appearing on the display, not the controller.

In the questionnaire, we used the term "the bear," not "Marching Bear," and we did not indicate whether the term "the bear" meant the virtual character or the robot controller. Then, after the experiment, we interviewed the participants in the small character group as to whether they felt the character on the display or the robot controller were cute. Most participants answered that the character on the display was cute and that they were not interested in the stuffed animal robot. Thus, these participants enjoyed using the Marching Bear but paid attention to the virtual character only.

However, participants in the large character group answered during the same interview that both were cute or that they could not choose one. Other interesting phenomena pertaining to this Marching Bear included the virtual character being able to walk based on the user's operational pace, e.g., when the user shook the robot's hands faster, the virtual character could walk faster. However, most participants operated this robot slowly, softly, and gently. These participants reported that they felt sorry for making the bear walk too fast or that it would hurt their arms or that it might be uncomfortable for this robot if they moved it too fast. We observed that one participant groomed the robot's coat, while another adjusted the ribbon on the robot's neck.

Therefore, most participants in the large character group thought that the Marching Bear was a companion or an independent character and that they had a strong emotional attachment to not only the virtual character but also to the stuffed animal robot.

\subsection{Intuitive Operation}

When we teach toddlers how to walk, we do not grab their legs directly because toddlers are not tools, and leading them by the hand to make a toddler walk is quite intuitive for us. However, the results of the experiment showed that both groups' participants first focused on moving the robot's legs to make the virtual character walk. This means that they intuitively thought that moving the robot's legs led to making the character walk. In fact, these behaviors indicated that the participants regarded this robot as just a tool at that time. When they actually moved the robot's legs directly to make the character walk, the robot could not keep a usual posture, e.g., this caused a head-down posture and they handled the robot too roughly. Therefore, participants thought that the Marching Bear was only a tool for the experimental setting.

However, after spending some time with the interactive system, the participants noticed how to make the Marching Bear walk, and then their behavior drastically changed. For example, some participants in the large character group moved the character so that it did not hit the walls in the virtual world. Eventually, these participants 
regarded the Marching Bear as a companion and independent character. Their behaviors became more gentle and sincere compared to the beginning of the experiment.

\section{Conclusions}

In this study, we developed the interface system, "Marching Bear," where the robot controller's motions are reflected on the motions of certain characters appearing on a computer display. We then conducted an experiment to investigate the effectiveness of the interface system as to whether or not this interface system was enjoyable. A questionnaire was used and an observation conducted as to whether or not users could move the robot's arms to make it walk. We also investigated the effectiveness of the interface system by using a large display and a smaller one. A virtual character was displayed on a laptop PC's display as part of a small character group, or it was displayed on an LCD monitor that enlarged the virtual character as part of a large character group.

The results of the experiments using these two groups demonstrated that the larger character had a much stronger effect on the participants' emotional aspect; they felt that the character had some emotions and that they could communicate with the character well. The robot controls of the interface system Marching Bear was not only enjoyable for the users, we felt that the bear could be a users' companion. The Marching Bear was a success in the sense that it got users interested in the controlling operations.

However, a few participants in the small character group felt effects similar to what most of the participants felt in the large character group, and a few participants in the large character group did not feel such effects. We speculate that the participants' individual differences would account for this phenomenon. Therefore, we are planning to investigate the effects of individual differences on emotional aspects when participants control the Marching Bear.

\section{References}

1. Wii Remote: http://wii.com/

2. TATACON (in Japanese), http://www.bandainamcogames.co.jp/donderpage/

3. EyeToy USB Camera: http://www.eyetoy.com/

4. Sekiguchi, D., Inami, M., Tachi, S.: RobotPHONE:RUI for Interpersonal Communication. In: The proceedings of CHI2001 Extended Abstracts, pp. 277-278 (2001)

5. Johnson, M.P., Wilson, A., Blumberg, B., Kline, C., Bobick, A.: Sympathetic Interface: Using a Plush Toy to Direct Synthetic Characters. In: The proceedings of CHI99, pp. 152-158 (1999)

6. Strommen, E.: When the Interface is a Talking Dinosaur: Leaning Across Media with ActiMates Barney. In: The proceedings of CHI98, pp. 288-295 (1998)

7. Koizumi, N., Shimizu, N., Sugimoto, M., Nii, H., Inami, M.: Development of Hand Puppet type Robotic User Interface. Journal of Virtual Reality Society of Japan 11(2), 265-274 (2006) (in Japanese)

8. Sekiguchi, D., Inami, M., Kawakami, N., Tachi, S.: The Design of Internet-Based RobotPHONE. In: The proceedings of the 14th International Conference on Artificial Reality and Tele-existence 2004, pp. 223-228 (2004) 